\title{
A Mini Review on Capillary Isoelectric Focusing-Mass Spectrometry for Top-Down Proteomics
}

\author{
Tian Xu and Liangliang Sun* \\ Department of Chemistry, Michigan State University, East Lansing, MI, United States
}

Mass spectrometry (MS)-based top-down proteomics (TDP) requires high-resolution separation of proteoforms before electrospray ionization (ESI)-MS and tandem mass spectrometry (MS/MS). Capillary isoelectric focusing (cIEF)-ESI-MS and MS/MS could be an ideal method for TDP because CIEF can enable separation of proteoforms based on their isoelectric points (pls) with ultra-high resolution. cIEF-ESI-MS has been well-recognized for protein characterization since 1990s. However, the widespread adoption of clEF-MS for the characterization of proteoforms had been impeded by several technical challenges, including the lack of highly sensitive and robust ESI interface for coupling CIEF to MS, ESI suppression of analytes from ampholytes, and the requirement of manual operations. In this mini review, we summarize the technical improvements of cIEF-ESI-MS for characterizing proteoforms and highlight

OPEN ACCESS

Edited by:

Alberto Salomone, University of Turin, Italy

Reviewed by:

Qiuling Zheng,

China Pharmaceutical

University, China

Fabio Di Nardo,

University of Turin, Italy

*Correspondence:

Liangliang Sun

Isun@chemistry.msu.edu

Specialty section: This article was submitted to

Analytical Chemistry,

a section of the journal

Frontiers in Chemistry

Received: 10 January 2021 Accepted: 16 March 2021

Published: 09 April 2021

Citation:

Xu T and Sun L (2021) A Mini Review on Capillary Isoelectric Focusing-Mass

Spectrometry for Top-Down

Proteomics. Front. Chem. 9:651757. doi: 10.3389/fchem.2021.651757 some recent applications to hydrophobic proteins, urinary albumin variants, charge variants of monoclonal antibodies, and large-scale TDP of complex proteomes.

Keywords: capillary isoelectric focusing-mass spectrometry, top-down proteomics, ESI interface, ampholytes, proteoform, monoclonal antibody, hydrophobic protein

\section{INTRODUCTION}

Top-down proteomics (TDP) aims to globally characterize proteoforms in cells. The concept of "proteoform" was published in 2013 to describe all the forms of protein molecules derived from a same gene on account of genetic variations, alternative splicing, and post-translational modifications (PTMs) (Smith et al., 2013). As proteoforms from a same gene can have divergent functions, characterization of proteomes in a proteoform-specific manner is imperative for understanding critical biological processes and disease mechanism (Ntai et al., 2018; Smith and Kelleher, 2018). TDP is ideal for characterization of proteoforms because it directly measures intact proteoforms using mass spectrometry (MS) and tandem mass spectrometry (MS/MS) for determining proteoforms' masses, sequences, and PTMs (Chen et al., 2017). Large-scale TDP of complex proteomes require sufficient liquid-phase separations of proteoforms prior to MS and MS/MS (Tran et al., 2011; Chen et al., 2017; Gomes and Yates, 2019; Schaffer et al., 2019; Shen et al., 2019).

Capillary isoelectric focusing (cIEF) separates amphoteric compounds (e.g., proteins) according to their isoelectric points (pIs) with the assistance of ampholytes (Righetti et al., 1997). cIEF can achieve ultrahigh-resolution separation of proteins with as low as $0.004 \mathrm{pI}$ differences (Shen et al., 1999; Kahle and Wätzig, 2018). Integrating cIEF with electrospray ionization (ESI)-MS is ideal for high-resolution separation and confident identification of proteoforms. cIEF-MS for protein characterization has been pioneered by Lee and Smith group in 1990s (Tang et al., 1995; Yang et al., 1998; Jensen et al., 1999; Paša-Tolić et al., 1999). 
However, for a long time, cIEF-ESI-MS suffered from low sensitivity due to the significant sample dilution by the sheath liquid in the coaxial sheath flow CE-MS interface (Smith et al., 1988) and the ionization suppression of analytes from ampholytes. The early cIEF-MS studies required manually pulling the separation capillary out of catholyte for focusing and inserting it into the ESI interface for mobilization and MS detection (Jensen et al., 1999; Paša-Tolić et al., 1999), impeding the widespread adoption of the technique for protein characterization.

During the last two decades, great efforts have been made for improving the sensitivity of cIEF-MS through developing new CE-MS interfaces and mitigating ampholyte impacts as well as for developing automated cIEF-MS methods. Several review papers focusing on cIEF-MS have been published recently (Silvertand et al., 2008; Hühner et al., 2015; Lechner et al., 2019). In this mini review, we summarize the important technical progress of cIEF-MS and highlight most recent applications of automated cIEF-MS for top-down MS characterization of hydrophobic proteins, urinary albumin variants, charge variants of monoclonal antibodies (mAbs), and large-scale TDP of complex proteomes.

\section{TECHNICAL DEVELOPMENT OF CIEF-MS}

\section{CE-MS Interface}

Online hyphenation of CIEF and ESI-MS requires a CEMS interface that can establish electrical continuity for
CE separation, and meanwhile produces stable electrospray. Sheath-flow interfaces are well-suited for cIEF-MS studies. Apart from assisting ionization, the sheath liquid can serve as the chemical mobilizer to facilitate protein mobilization after cIEF focusing. The concentration of carrier ampholytes can be significantly decreased after mixing cIEF effluent with the sheath liquid, thus benefiting ESI-MS detection. The coaxial sheath flow interface (Smith et al., 1988), designed by Smith group in 1988, is the earliest version of interface used for cIEFMS (Figure 1A). However, significant sample dilution can occur using this interface due to the much higher flow rate of the sheath liquid compared to the sample flow in CE capillary $(1-10 \mu \mathrm{L} / \mathrm{min}$ vs. low $\mathrm{nL} / \mathrm{min}$ ).

Tremendous efforts have been invested to improve the sensitivity of sheath-flow interface by reducing the flow rate of sheath liquid to the $\mathrm{nL} / \mathrm{min}$ level. The Chen group constructed a flow-through microvial interface by placing the separation capillary in a stainless-steel emitter (Maxwell et al., 2010) (Figure 1B). The sheath buffer is delivered through the gap between the capillary and the emitter via a springe pump at a flow rate of $100-300 \mathrm{~nL} / \mathrm{min}$. They achieved at least fivetimes better limit of detections (LODs) for amino acids using the interface compared to the coaxial sheath flow interface. The Dovichi group introduced an electrokinetically pumped sheath flow interface to the field in 2010 (Wojcik et al., 2010). The interface employed electroosmotic flow in the glass spray emitter to pump the sheath liquid at the $\mathrm{nL} / \mathrm{min}$ level for ESI (Figure 1C). The interface was further improved regarding sensitivity and
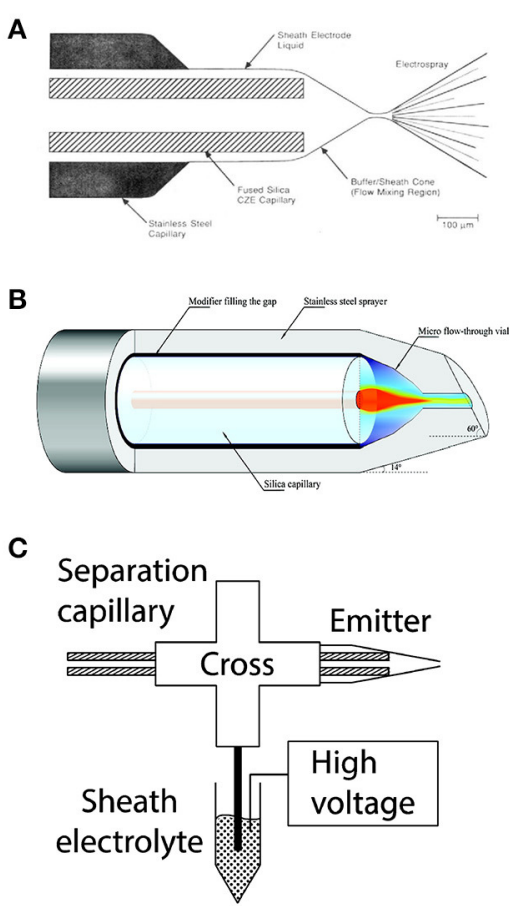

D

Step 1: Inject catholyte plug

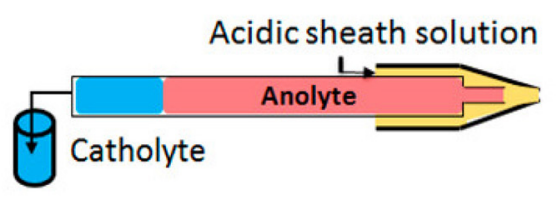

Step 2: Inject protein sample (in ampholytes)

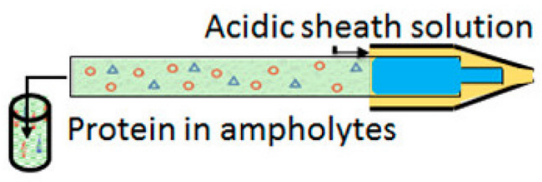

Step 3: Focusing \& mobilization

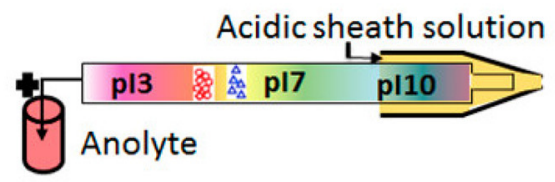

FIGURE 1 | (A) Coaxial sheath flow interface. Reproduced with permission from Smith et al. (1988). Copyright 1988 American Chemical Society. (B) Flow-through microvial interface. Reproduced with permission from Zhong et al. (2011a). Copyright 2011 American Chemical Society. (C) Electrokinetically pumped sheath flow interface. Reproduced with permission from Sun et al. (2015). Copyright 2015 American Chemical Society. (D) Flowchart of automated clEF-MS. Reproduced with permission from Dai et al. (2018). Copyright 2018 American Chemical Society. 
robustness by adjusting the emitter orifice size and distance between the capillary end and emitter orifice in 2013 and 2015 (Sun et al., 2013, 2015). The improved interface-based CE-MS showed extremely high sensitivity for peptides and proteoforms (Sun et al., 2013; Yang et al., 2018; Lubeckyj et al., 2019). The electrokinetically pumped sheath flow CE-MS interface has been commercialized by the CMP Scientific (https://www. cmpscientific.com/) as the EMASS-II CE-MS Ion Source. Both the two sheath-flow interfaces have been successfully applied to cIEF-MS characterization of intact proteins (Zhong et al., 2011a,b; Zhu et al., 2017). More recently, several other sheathflow CE-MS interfaces with $\mathrm{nL} / \mathrm{min}$ flow rates of sheath liquid have been developed (Choi et al., 2016; Fang et al., 2018; Krenkova et al., 2019; Höcker et al., 2020). However, based on our best knowledge, there are still no literature reports about using these new CE-MS interfaces for cIEF-MS.

\section{Reducing the Impact of Carrier Ampholytes}

Carrier ampholytes induce ionization suppression of analytes (Dai et al., 2018), restricting the overall sensitivity of cIEFMS. However, they are indispensable for establishing and maintaining $\mathrm{pH}$ gradient needed for cIEF separation. Reducing the concentration of ampholyte can significantly improve MS signal, but it adversely impacts separation resolution. Compromise has to be made between MS signal and separation resolution when performing CIEF-MS analysis and the concentration of ampholytes needs to be reduced to $0.5 \%$ or even lower (Paša-Tolić et al., 1999; Hühner et al., 2015; Zhu et al., 2017).

Besides decreasing the ampholyte concentration, several other approaches have been validated for reducing the impact of carrier ampholytes on ESI of analytes. First, integrating the cIEF with microdialysis (MD) is effective to remove the low-molecularweight ampholytes from relatively large proteins before ESI (Lamoree et al., 1997). The method is not widely used due to reduced separation efficiency caused by the MD devices. Second, cIEF-MS has been performed in a carrier ampholyte-free condition (Zhu et al., 2012). As amino acids have amphoteric properties and are much smaller than peptides and proteins, they can be used for establishing $\mathrm{pH}$ gradient for cIEF separation. The drawback is amino acids cannot establish a continuous $\mathrm{pH}$ gradient in the separation capillary as commercialized carrier ampholytes. Third, some work has been successfully done for creating an immobilized $\mathrm{pH}$ gradient in cIEF capillaries by covalently immobilizing carrier ampholytes onto in-situ formed monolithic materials for protein separation (Zhu et al., 2006; Yang et al., 2010; Liang et al., 2011; Liu et al., 2019). However, more systematic investigations of the immobilized $\mathrm{pH}$ gradient cIEF-MS needs to be done before deploying it in routine protein characterization.

\section{Development of Automated cIEF-MS}

The cIEF-MS analyses typically were implemented in a semi-online manner, where capillary outlet was inserted in a catholyte reservoir with basic buffer for focusing, and then manually transferred to an interface filled with acidic sheath liquid for mobilization and ionization (Jensen et al., 1999). Alternatively, the capillary was fixed in the interface during the whole process and sheath liquid in the interface was substituted from basic buffer to acidic sheath liquid when focusing was completed (Wang et al., 2018). The appearance of "sandwich" injection configuration in 2009 makes it possible to perform fully automated cIEF-MS analysis (Mokaddem et al., 2009). The method was carried out by filling the capillary with MS compatible catholyte buffer such as ammonia hydroxide, followed by a plug of sample-ampholyte mixture. Thus, the cIEF focusing could be facilitated after applying voltage even though its outlet was installed in an interface with acidic sheath liquid. After focusing, a low pressure (i.e., 50 mbar) or chemical mobilization was employed to drive focused proteins toward MS for detection. The chemical mobilization can automatically be initiated when cations from anolyte and anions from sheath liquid enter the capillary and gradually disrupt the $\mathrm{pH}$ gradient. The automated cIEF-MS method is an appealing technique for various applications.

\section{APPLICATIONS}

Because of the drastic improvement in the CE-MS interface regarding stability and sensitivity, the method for reducing the negative influence of ampholytes on ESI-MS, and the automated operation, cIEF-MS has been recognized as a powerful and sensitive analytical tool for top-down MS characterization of intact proteins.

\section{Automated cIEF-ESI-MS for Top-Down Characterization of Hydrophobic Proteins, Urinary Albumin, and mAb Charge Variants}

Characterization of hydrophobic proteins with CE-MS is always challenging because additives for stabilizing hydrophobic proteins such as thiourea, urea, and surfactants are not compatible with MS. Mokaddem et al. performed automated cIEF-ESI-MS analysis of a mixture of hydrophobic and hydrophilic proteins in a glycerol-water medium with a commercialized coaxial interface (Mokaddem et al., 2009). The automated cIEF-MS was carried out in three steps (Figure 1D). First, the capillary $(100 \mathrm{~cm})$ was injected with a plug of catholyte $(60 \mathrm{~cm})$ and a plug of sample-ampholyte mixture $(40 \mathrm{~cm})$. Then, a voltage $(30 \mathrm{kV})$ was applied on the capillary to facilitate protein focusing. Finally, after focusing completed, a pressure (50 mbar) was applied on the capillary to mobilize protein bands. The study found that glycerol in the concentration range of $10-30 \%(\mathrm{v} / \mathrm{v})$ was both MS compatible and well-preserved protein solubility. The data indicate the potential of cIEF-MS for the characterization of membrane proteins. The method was later employed by Lecoeur et al. for qualitative and quantitative analysis of hydrophobic and hydrophilic whey proteins in bovine milk (Lecoeur et al., 2010).

The automated cIEF-MS with glycerol medium is also wellsuited for top-down characterization of mAb charge variants. Dai 
et al. developed an automated cIEF-MS method using the similar "sandwich" injection method reported by Mokaddem et al., a capillary with neutral coating, and an electrokinetically pumped sheath flow interface (Dai et al., 2018). Using this method, the charge variants of various mAbs, including trastuzumab, bevacizumab, infliximab, and cetuximab, were well-resolved. The separation results showed good correlation with that from cIEFUV analysis. Later, Dai et al. created a middle-up approach based on the automated cIEF-MS to boost its performance for delineating complex mAb charge variants (i.e., cetuximab), leading to the identification of at least eight different charge variants of cetuximab (Dai and Zhang, 2018). More recently, Tie et al. applied the automated cIEF-MS to the characterization of urinary albumin species from the membranous nephropathy (MN) patients (Tie et al., 2020). They observed distinct patterns of urinary albumin charge variants from the primary and secondary MN samples, suggesting the potential of the technique for distinguishing different subtypes of MN.

Wang et al. demonstrated top-down characterization of $\mathrm{mAb}$ charge variants on an automated cIEF-ESI-QTOF MS system with a flow through microvial interface (Wang et al., 2018; Wang and Chen, 2019). With consumption of only $30 \mathrm{ng}$ of infliximab, four charge variants with $0.05-0.2 \mathrm{pI}$ differences and 13 glycoforms were detected (Wang et al., 2018). Developing microchip-based cIEF-MS is also very attractive to pharmaceutical industry. Recent microchip-based cIEF-MS system developed by Mack et al. provided real-time optical monitoring of focusing and mobilization process of cIEF, good resolving power and high throughput ( $15 \mathrm{~min}$ each assay) for characterization of mAb charge variants (Mack et al., 2019). Besides a direct coupling of cIEF and ESI-MS, cIEF has also been coupled to CZE-MS using a mechanical valve or a nanoliter valve for high-resolution characterization of intact proteins and $\mathrm{mAb}$ charge variants (Hühner et al., 2017; Montealegre and Neusüß, 2018).

These works suggest automated cIEF-MS is a promising tool for quality control of therapeutic mAbs regarding charge variants and PTMs in pharmaceuticals by providing high-resolution separation and accurate mass determination. All the cIEF-MS studies mentioned here only performed protein mass measurement without MS/MS. More efforts need to be made about integrating extensive gas-phase fragmentation techniques with automated cIEF-MS for delineation of proteoforms.

\section{Automated cIEF-ESI-MS/MS for Large-Scale TDP}

Coupling automated cIEF-MS with MS/MS enables online fragmentation of separated proteoforms for identifying proteoform sequences and localizing PTMs. Our lab presented the first work of automated cIEF-MS/MS for large-scale TDP of complex proteomes in 2020 (Xu et al., 2020). The automated cIEF-MS/MS platform was constructed by integrating a linearpolyacrylamide (LPA) coated capillary with an Orbitrap mass spectrometer via an electrokinetically pumped sheath flow interface. Based on "sandwich" injection configuration and chemical mobilization, automated cIEF-MS/MS methods identified $711 \mathrm{E}$. coli proteoforms in a single run by consuming only nanograms of proteins.

Furthermore, combining size exclusion chromatography (SEC) and the automated cIEF-MS/MS identified nearly 2000 proteoforms from the E. coli proteome. SEC-cIEF-MS/MS was further employed for label-free quantitative TDP of male and female zebrafish brains (Figure 2). Thousands of proteoforms were quantified, and 263 proteoforms showed statistically significant difference in abundance between male and female zebrafish brains. Gene ontology analysis found many of these differentially expressed proteoforms were associated with neuronal development and their expression can be regulated by hormones, disclosing the sex dimorphism of zebrafish brains at the proteoform level. The work clearly demonstrates the capability of automated cIEF-MS/MS for large-scale qualitative and quantitative TDP.

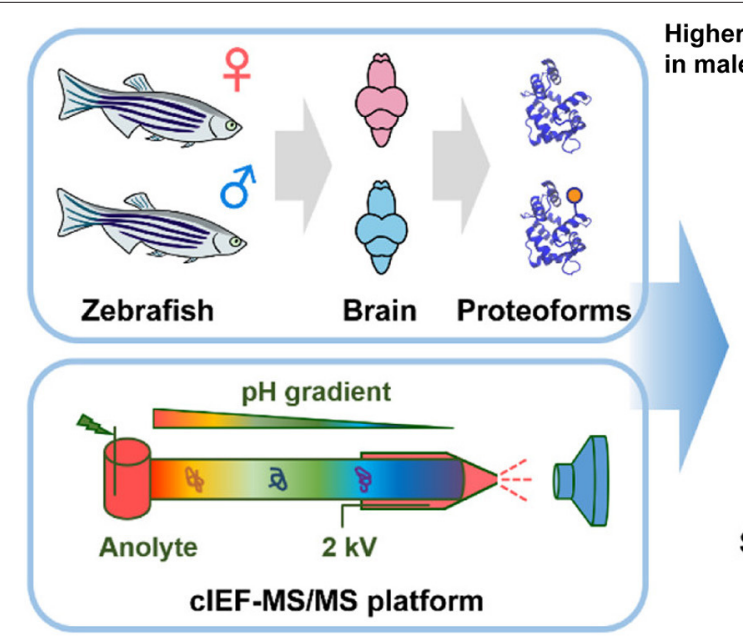

FIGURE 2 | Flow chart of label-free quantitative TDP of female and male zebrafish brains using automated cIEF-MS/MS. Reproduced with permission from Xu et al. (2020). Copyright 2020 American Chemical Society. 


\section{DISCUSSION}

Reversed-phase liquid chromatography (RPLC)-MS/MS is the routine choice for top-down characterization of proteoforms (Tran et al., 2011; Chen et al., 2017; Schaffer et al., 2019). However, RPLC typically fails to separate proteoforms, especially large proteoforms (i.e., larger than $30 \mathrm{kDa}$ ), with high separation efficiency due to their low diffusion coefficients and strong interactions with the reversed-phase beads. Capillary zone electrophoresis (CZE)-MS/MS has been suggested as a useful alternative for TDP due to high separation efficiency of CZE for proteoforms according to their electrophoretic mobilities and high sensitivity of CZE-MS for proteoform measurements (Gomes and Yates, 2019; Shen et al., 2019). Although submicroliter sample loading volumes have been reported for TDP using CZE-MS/MS (Lubeckyj et al., 2017, 2019), the loading volume is still limited to a small portion of the total capillary volume for maintaining high separation efficiency. cIEF-MS/MS has shown high potential for advancing TDP because cIEF can achieve proteoform separations with high resolution and has much higher sample loading capacity compared to CZE.

cIEF-MS has become an important technique for top-down MS characterization of proteins. However, it still needs to overcome several technical challenges. First, current cIEF-MS methods can hardly make full use of high resolving power of cIEF. Decreasing concentration of carrier ampholytes is a common choice to enhance sensitivity, but it can cause reduced separation resolution. Immobilized $\mathrm{pH}$ gradient cIEF could be an alternative

\section{REFERENCES}

Chen, B., Brown, K. A., Lin, Z., and Ge, Y. (2017). Top-down proteomics: ready for prime time? Anal. Chem. 90, 110-127. doi: 10.1021/acs.analchem. $7 \mathrm{~b} 04747$

Choi, S. B., Zamarbide, M., Manzini, M. C., and Nemes, P. (2016). Tapered-tip capillary electrophoresis nano-electrospray ionization mass spectrometry for ultrasensitive proteomics: the mouse cortex. J. Am. Soc. Mass Spectrom. 28, 597-607. doi: 10.1007/s13361-016-1532-8

Dai, J., Lamp, J., Xia, Q., and Zhang, Y. (2018). Capillary isoelectric focusingmass spectrometry method for the separation and online characterization of intact monoclonal antibody charge variants. Anal. Chem. 90, 2246-2254. doi: 10.1021/acs.analchem.7b04608

Dai, J., and Zhang, Y. (2018). A middle-up approach with online capillary isoelectric focusing/mass spectrometry for in-depth characterization of cetuximab charge heterogeneity. Anal. Chem. 90, 14527-14534. doi: 10.1021/acs.analchem.8b04396

Fang, P., Pan, J. Z., and Fang, Q. (2018). A robust and extendable sheath flow interface with minimal dead volume for coupling CE with ESI-MS. Talanta 180, 376-382. doi: 10.1016/j.talanta.2017.12.046

Gomes, F. P., and Yates, I. I. I., J. R. (2019). Recent trends of capillary electrophoresis-mass spectrometry in proteomics research. Mass Spectrom. Rev. 38, 445-460. doi: 10.1002/mas.21599

Höcker, O., Knierman, M., Meixner, J., and Neusüß, C. (2020). Two capillary approach for a multifunctional nanoflow sheath liquid interface for capillary electrophoresis-mass spectrometry. Electrophoresis 42, 369-373. doi: 10.1002/elps.202000169

Hühner, J., Jooß, K., and Neusüß, C. (2017). Interference-free mass spectrometric detection of capillary isoelectric focused proteins, including charge variants of a model monoclonal antibody. Electrophoresis 38, 914-921. doi: 10.1002/elps.201600457 because it allows cIEF separation without carrier ampholytes and has shown good separation efficiency of proteins with UV detection. However, its applications in cIEF-MS still need to be systematically evaluated. Second, cIEF-MS suffers from limited lifetime of capillary coating. cIEF-MS studies generally use ammonia hydroxide $(\mathrm{pH}>11)$ as catholyte. However, the most commonly used LPA coating cannot stand this high $\mathrm{pH}$ for a long time. Using a catholyte with $\mathrm{pH}$ lower than 10 can improve the stability of capillary coating (Ramsay et al., 2011). Exploring novel capillary coatings that are stable at high $\mathrm{pH}$ is vital for reproducible and robust cIEF-MS analyses. Third, analysis of highly basic $(\mathrm{pI}>10)$ or acidic $(\mathrm{pI}<3)$ proteoforms remains difficult for cIEF as the commonly used carrier ampholytes cover a pI range of 3-10. Thus, novel cIEF-MS methods are required for characterizing proteoforms with extremely low or high pIs.

\section{AUTHOR CONTRIBUTIONS}

TX and LS wrote the manuscript and made comments. Both authors contributed to the article and approved the submitted version.

\section{FUNDING}

We thank the support from the National Science Foundation (CAREER Award, Grant DBI1846913) and the National Institutes of Health (Grant R01GM125991).
Hühner, J., Lämmerhofer, M., and Neusüß, C. (2015). Capillary isoelectric focusing-mass spectrometry: coupling strategies and applications. Electrophoresis 36, 2670-2686. doi: 10.1002/elps.2015 00185

Jensen, P. K., Paša-Tolić, L., Anderson, G. A., Horner, J. A., Lipton, M. S., Bruce, J. E., et al. (1999). Probing proteomes using capillary isoelectric focusing-electrospray ionization Fourier transform ion cyclotron resonance mass spectrometry. Anal. Chem. 71, 2076-2084. doi: 10.1021/ ac990196p

Kahle, J., and Wätzig, H. (2018). Determination of protein charge variants with (imaged) capillary isoelectric focusing and capillary zone electrophoresis. Electrophoresis 39, 2492-2511. doi: 10.1002/elps.201800079

Krenkova, J., Kleparnik, K., Luksch, J., and Foret, F. (2019). Microfabricated liquid junction hybrid capillary electrophoresis-mass spectrometry interface for fully automated operation. Electrophoresis 40, 2263-2270. doi: 10.1002/elps.201900049

Lamoree, M., Tjaden, U., and Van Der Greef, J. (1997). Use of microdialysis for the on-line coupling of capillary isoelectric focusing with electrospray mass spectrometry. J. Chromatogr. A 777, 31-39. doi: 10.1016/S0021-9673(97)00430-5

Lechner, A., Giorgetti, J., Gahoual, R., Beck, A., Leize-Wagner, E., and François, Y. N. (2019). Insights from capillary electrophoresis approaches for characterization of monoclonal antibodies and antibody drug conjugates in the period 2016-2018. J. Chromatogr. B 1122, 1-17. doi: 10.1016/j.jchromb.2019.05.014

Lecoeur, M., Gareil, P., and Varenne, A. (2010). Separation and quantitation of milk whey proteins of close isoelectric points by on-line capillary isoelectric focusing-Electrospray ionization mass spectrometry in glycerol-water media. $J$. Chromatogr. A 1217, 7293-7301. doi: 10.1016/j.chroma.2010.09.043

Liang, Y., Zhu, G., Wang, T., Zhang, X., Liang, Z., Zhang, L., et al. (2011). Fast preparation of monolithic immobilized $\mathrm{pH}$ gradient 
column by photopolymerization and photografting techniques for isoelectric focusing separation of proteins. Electrophoresis 32, 2911-2914. doi: 10.1002/elps.201100195

Liu, R., Cheddah, S., Liu, S., Liu, Y., Wang, Y., and Yan, C. (2019). A porous layer open-tubular capillary column with immobilized $\mathrm{pH}$ gradient (PLOTIPG) for isoelectric focusing of amino acids and proteins. Anal. Chim. Acta 1048, 204-211. doi: 10.1016/j.aca.2018.10.036

Lubeckyj, R. A., Basharat, A. R., Shen, X., Liu, X., and Sun, L. (2019). Large-scale qualitative and quantitative top-down proteomics using capillary zone electrophoresis-electrospray ionization-tandem mass spectrometry with nanograms of proteome samples. J. Am. Soc. Mass Spectrom. 30, 1435-1445. doi: 10.1007/s13361-019-02167-w

Lubeckyj, R. A., McCool, E. N., Shen, X., Kou, Q., Liu, X., and Sun, L. (2017). Single-shot top-down proteomics with capillary zone electrophoresiselectrospray ionization-tandem mass spectrometry for identification of nearly 600 escherichia coli proteoforms. Anal. Chem. 89, 12059-12067. doi: 10.1021/acs.analchem.7b02532

Mack, S., Arnold, D., Bogdan, G., Bousse, L., Danan, L., Dolnik, V., et al. (2019). A novel microchip-based imaged CIEF-MS system for comprehensive characterization and identification of biopharmaceutical charge variants. Electrophoresis 40, 3084-3091. doi: 10.1002/elps.201900325

Maxwell, E. J., Zhong, X., Zhang, H., Van Zeijl, N., and Chen, D. D. (2010). Decoupling CE and ESI for a more robust interface with MS. Electrophoresis 31, 1130-1137. doi: 10.1002/elps.200900517

Mokaddem, M., Gareil, P., and Varenne, A. (2009). Online CIEF-ESI-MS in glycerol-water media with a view to hydrophobic protein applications. Electrophoresis 30, 4040-4048. doi: 10.1002/elps.200900091

Montealegre, C., and Neusüß, C. (2018). Coupling imaged capillary isoelectric focusing with mass spectrometry using a nanoliter valve. Electrophoresis 39, 1151-1154. doi: 10.1002/elps.201800013

Ntai, I., Fornelli, L., Dehart, C. J., Hutton, J. E., Doubleday, P. F., Leduc, R. D., et al. (2018). Precise characterization of KRAS4b proteoforms in human colorectal cells and tumors reveals mutation/modification cross-talk. Proc. Natl. Acad. Sci. U.S.A. 115, 4140-4145. doi: 10.1073/pnas.1716122115

Paša-Tolić, L., Jensen, P. K., Anderson, G. A., Lipton, M. S., Peden, K. K., Martinović, S., et al. (1999). High throughput proteome-wide precision measurements of protein expression using mass spectrometry. J. Am. Chem. Soc. 121, 7949-7950. doi: 10.1021/ja991063o

Ramsay, L. M., Cermak, N., Dada, O. O., and Dovichi, N. J. (2011). Capillary isoelectric focusing with $\mathrm{pH} 9.7$ cathode for the analysis of gastric biopsies. Anal. Bioanal. Chem. 400, 2025-2030. doi: 10.1007/s00216-011-4926-2

Righetti, P. G., Gelfi, C., and Conti, M. (1997). Current trends in capillary isoelectric focusing of proteins. J. Chromatogr. B Biomed. Appl. 699, 91-104. doi: 10.1016/S0378-4347(96)00208-3

Schaffer, L. V., Millikin, R. J., Miller, R. M., Anderson, L. C., Fellers, R. T., Ge, Y., et al. (2019). Identification and quantification of proteoforms by mass spectrometry. Proteomics 19:1800361. doi: 10.1002/pmic.201800361

Shen, X., Yang, Z., Mccool, E. N., Lubeckyj, R. A., Chen, D., and Sun, L. (2019). Capillary zone electrophoresis-mass spectrometry for top-down proteomics. TrAC Trends Anal. Chem. 120:115644. doi: 10.1016/j.trac.2019.115644

Shen, Y., Xiang, F., Veenstra, T. D., Fung, E. N., and Smith, R. D. (1999). Highresolution capillary isoelectric focusing of complex protein mixtures from lysates of microorganisms. Anal. Chem. 71, 5348-5353. doi: 10.1021/ac9909305

Silvertand, L., Torano, J. S., Van Bennekom, W., and De Jong, G. (2008). Recent developments in capillary isoelectric focusing. J. Chromatogr. A 1204, 157-170. doi: 10.1016/j.chroma.2008.05.057

Smith, L. M., and Kelleher, N. L. (2018). Proteoforms as the next proteomics currency. Science 359, 1106-1107. doi: 10.1126/science.aat1884

Smith, L. M., Kelleher, N. L., Linial, M., Goodlett, D., Langridge-Smith, P., Goo, Y. A., et al. (2013). Proteoform: a single term describing protein complexity. Nat. Methods 10, 186-187. doi: 10.1038/nmeth.2369

Smith, R. D., Barinaga, C. J., and Udseth, H. R. (1988). Improved electrospray ionization interface for capillary zone electrophoresis-mass spectrometry. Anal. Chem. 60, 1948-1952. doi: 10.1021/ac00169a022

Sun, L., Zhu, G., Zhang, Z., Mou, S., and Dovichi, N. J. (2015). Third-generation electrokinetically pumped sheath-flow nanospray interface with improved stability and sensitivity for automated capillary zone electrophoresis-mass spectrometry analysis of complex proteome digests. J. Proteome Res. 14, 2312-2321. doi: 10.1021/acs.jproteome.5b00100
Sun, L., Zhu, G., Zhao, Y., Yan, X., Mou, S., and Dovichi, N. J. (2013). Ultrasensitive and fast bottom-up analysis of femtogram amounts of complex proteome digests. Angew. Chem. Int. Ed. 52, 13661-13664. doi: 10.1002/anie.201308139

Tang, Q., Harrata, A. K., and Lee, C. S. (1995). Capillary isoelectric focusingelectrospray mass spectrometry for protein analysis. Anal. Chem. 67, 3515-3519. doi: 10.1021/ac00115a021

Tie, C., Liu, L., Feng, T., Sa, R., Xia, Q., Liang, H., et al. (2020). Differential analysis of urinary albumin for membranous nephropathy patients by online capillary isoelectric focusing-Mass spectrometry. J. Proteom. 216:103676. doi: 10.1016/j.jprot.2020.103676

Tran, J. C., Zamdborg, L., Ahlf, D. R., Lee, J. E., Catherman, A. D., Durbin, K. R., et al. (2011). Mapping intact protein isoforms in discovery mode using top-down proteomics. Nature 480, 254-258. doi: 10.1038/nature10575

Wang, L., Bo, T., Zhang, Z., Wang, G., Tong, W., and Chen, D. D. (2018). High resolution capillary isoelectric focusing mass spectrometry analysis of peptides, proteins, and monoclonal antibodies with a flow-through microvial interface. Anal. Chem. 90, 9495-9503. doi: 10.1021/acs.analchem.8b02175

Wang, L., and Chen, D. D. (2019). Analysis of four therapeutic monoclonal antibodies by online capillary isoelectric focusing directly coupled to quadrupole time-of-flight mass spectrometry. Electrophoresis 40, 2899-2907. doi: 10.1002/elps.201900195

Wojcik, R., Dada, O. O., Sadilek, M., and Dovichi, N. J. (2010). Simplified capillary electrophoresis nanospray sheath-flow interface for high efficiency and sensitive peptide analysis. Rapid Commun. Mass Spectrom. 24, 2554-2560. doi: $10.1002 / \mathrm{rcm} .4672$

Xu, T., Shen, X., Yang, Z., Chen, D., Lubeckyj, R. A., Mccool, E. N., et al. (2020). Automated capillary isoelectric focusing-tandem mass spectrometry for qualitative and quantitative top-down proteomics. Anal. Chem. 92, 15890-15898. doi: 10.1021/acs.analchem.0c03266

Yang, C., Wang, S., Chang, C., Wang, Y., and Hu, X. (2010). Capillary isoelectric focusing with an open tubular immobilized $\mathrm{pH}$ gradient. Anal. Chem. 82, 1580-1583. doi: 10.1021/ac902223y

Yang, L., Lee, C. S., Hofstadler, S. A., Pasa-Tolic, L., and Smith, R. D. (1998). Capillary isoelectric focusing-electrospray ionization Fourier transform ion cyclotron resonance mass spectrometry for protein characterization. Anal. Chem. 70, 3235-3241. doi: 10.1021/ac980224o

Yang, Z., Shen, X., Chen, D., and Sun, L. (2018). Microscale reversed-phase liquid chromatography/capillary zone electrophoresis-tandem mass spectrometry for deep and highly sensitive bottom-up proteomics: identification of 7500 proteins with five micrograms of an MCF7 proteome digest. Anal. Chem. 90, 10479-10486. doi: 10.1021/acs.analchem.8b02466

Zhong, X., Maxwell, E. J., and Chen, D. D. (2011a). Mass transport in a micro flow-through vial of a junction-at-the-tip capillary electrophoresis-mass spectrometry interface. Anal. Chem. 83, 4916-4923. doi: 10.1021/ac200636y

Zhong, X., Maxwell, E. J., Ratnayake, C., Mack, S., and Chen, D. D. (2011b). Flow-through microvial facilitating interface of capillary isoelectric focusing and electrospray ionization mass spectrometry. Anal. Chem. 83, 8748-8755. doi: $10.1021 /$ ac202130f

Zhu, G., Sun, L., and Dovichi, N. J. (2017). Simplified capillary isoelectric focusing with chemical mobilization for intact protein analysis. J. Sep. Sci. 40, 948-953. doi: 10.1002/jssc.201601051

Zhu, G., Sun, L., Yang, P., and Dovichi, N. J. (2012). On-line amino acid-based capillary isoelectric focusing-ESI-MS/MS for protein digests analysis. Anal. Chim. Acta 750, 207-211. doi: 10.1016/j.aca.2012.04.026

Zhu, G., Yuan, H., Zhao, P., Zhang, L., Liang, Z., Zhang, W., et al. (2006). Macroporous polyacrylamide-based monolithic column with immobilized pH gradient for protein analysis. Electrophoresis 27, 3578-3583. doi: 10.1002/elps.200600189

Conflict of Interest: The authors declare that the research was conducted in the absence of any commercial or financial relationships that could be construed as a potential conflict of interest.

Copyright $\odot 2021 \mathrm{Xu}$ and Sun. This is an open-access article distributed under the terms of the Creative Commons Attribution License (CC BY). The use, distribution or reproduction in other forums is permitted, provided the original author(s) and the copyright owner(s) are credited and that the original publication in this journal is cited, in accordance with accepted academic practice. No use, distribution or reproduction is permitted which does not comply with these terms. 